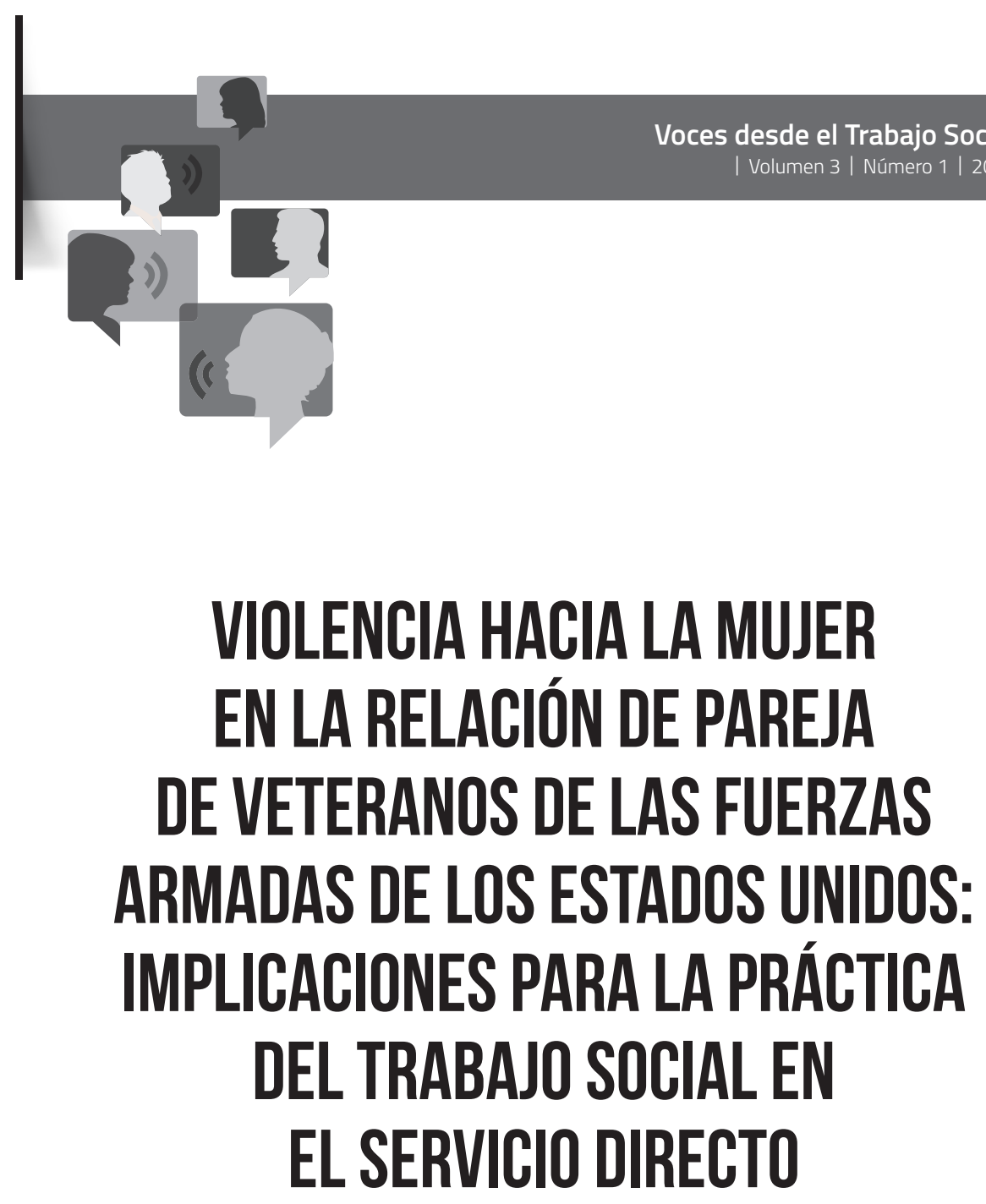




\section{Antonio Fernández-Lizardi}

Posee un Juris Doctor de la Universidad de Puerto Rico y una Maestría en Trabajo Social de la Universidad Interamericana. Es trabajador social clínico en el Hospital de Veteranos y abogado-notario en la práctica privada en Puerto Rico.

\section{Recibido:}

13 de febrero de 2015

\section{Aprobado:}

19 de septiembre de 2015 


\section{VIOLENCIA HACIA LA MUJER EN LA RELACIÓN DE PAREJA DE VETERANOS DE LAS FUERZAS ARMADAS DE LOS ESTADOS UNIDOS: IMPLICACIONES PARA LA PRÁCTICA DEL TRABAJO SOCIAL EN EL SERVICIO DIRECTO}

Antonio Fernández-Lizardi

Resumen

El objetivo del presente trabajo es estudiar, mediante una investigación documental, el tema de la violencia doméstica centrándonos en la población de hombres veteranos posterior a su reinserción a la vida civil. A su vez, se analizan las implicaciones de este problema para la práctica directa en trabajo social. Se pretende realizar una aportación a la conceptuación del problema y ofrecer recomendaciones de tipo metodológicas a la literatura profesional del trabajo social en Puerto Rico.

Descriptores: Violencia doméstica, despliegue, veteranos, salud mental, práctica de trabajo social. 


\section{Abstract}

The objective of this article is to study domestic violence, focusing on veteran males after their reinsertion in civilian life. The author analyzes the implications of this problem for social work practice and education in Puerto Rico and presents methodological recommendations.

Keywords: domestic violence, deployment, veterans, mental health and social work practice. 


\section{Introducción}

El tema de interés del presente trabajo es la violencia hacia la mujer en la relación de pareja de veteranos de las fuerzas armadas de los Estados Unidos y las implicaciones para la práctica del trabajo social en el servicio directo. Para efectos de esta investigación, violencia doméstica se refiere a los actos de agresión, física, verbal, emocional, sexual y restricción de la libertad, ejercidos por veteranos varones heterosexuales hacia las mujeres con quienes mantienen una relación de pareja. La violencia en la relación de pareja ha sido descrita históricamente de diversas maneras por diferentes autores de acuerdo a su perspectiva ideológica o teórica (Corsi, 1995; Burgos, 2002; Valle Ferrer, 2006; Maschi, 2009). Bradley (2009) define la violencia doméstica como un "patrón de control coercitivo en el cual el agresor ejerce poder hacia su víctima a través de amenazas verbales y violencia física" (p. 97). En esta definición se visualizan las diversas manifestaciones de la violencia como instrumentos o medios del agresor para obtener poder sobre su víctima.

La literatura profesional contiene diversidad de trabajos de investigación, conceptuación y proposiciones metodológicas que estudian la violencia en la pareja (Burgos, 2002; Valle Ferrer, 2006, 2007). No obstante, el énfasis mayor está en la víctima o sobreviviente. Aunque en alguna medida se aborda la violencia doméstica y la intervención con el agresor, no se hace con la misma intensidad, frecuencia o perspectiva que se hace con la situación de la víctima o sobreviviente. En Puerto Rico se han establecido programas de reeducación para el hombre agresor, pero muy pocos están dirigidos o conceptualizados desde la perspectiva del trabajo social. La mayoría de los trabajos sobre los hombres agresores en Puerto Rico, realizados por trabajadores sociales, no abordan la violencia doméstica del veterano y como resultado no 
están dirigidos a esta población (Molina \& Fernández, 2009; García-Toro, 2009; Molina, Fernández \& Berrios, 2015).

Este trabajo analiza el tema de la violencia doméstica, dando énfasis al hombre agresor, veterano de las fuerzas armadas de los Estados Unidos que participó en prácticas bélicas y retorna a su hogar y a la vida civil. A su vez, el trabajo pretende analizar las implicaciones de este problema para la práctica directa en trabajo social. Se pretende realizar una aportación a la conceptualización del problema y aportar recomendaciones de tipo metodológicas a la literatura profesional del trabajo social en Puerto Rico.

Para mayor claridad conceptual es pertinente definir el concepto veterano. La palabra veterano proviene de la palabra vetus, del latín que significa 'viejo'. El término también se utiliza para describir a una persona que tenga experiencia en un área u organización o que haya servido en las fuerzas armadas (Webster's New World Dictionary and Thesaurus, p. 684). Para efectos del presente trabajo un veterano es una persona que haya servido activamente en alguna rama de las fuerzas armadas de los Estados Unidos, entiéndase Ejército, Marina, Fuerza Aérea, Infantería Marina y Guarda Costanera y que su cumplimiento con dicho servicio haya sido, en caso de ser retirado, honorable.

Según la Ley 54 de 1989 de Puerto Rico, conocida como la Ley para la Prevención e Intervención con la Violencia Doméstica se considera violencia doméstica:

Un patrón de conducta constante de empleo de fuerza física o de violencia sicológica, intimidación o persecución contra una persona por parte de su cónyuge, ex-cónyuge, de la persona con quien cohabite o haya cohabitado, o con quien sostenga o haya 
sostenido una relación consensual, o con quien haya procreado hijos, cuyo resultado es daño físico a la persona agredida, a sus bienes o a cualquier otra persona con el propósito de causarle a aquélla grave daño emocional. (Ley 54, 1989, p.4)

En la búsqueda de literatura profesional de trabajo social en Puerto Rico, no se encontró ningún artículo profesional, ni tesis doctoral, escrito por trabajadores sociales en el servicio directo que abordara el tema de los veteranos con situaciones de violencia doméstica. Esta investigación es importante para la práctica de trabajo social en Puerto Rico porque enfoca una población en situaciones de opresión y con factores de vulnerabilidad, los cuales no han sido lo suficientemente abordados en la literatura del trabajo social con enfoque en servicios directos en Puerto Rico.

\section{Definición del Problema y Justificación de la Importancia del Tema para el Trabajo Social en el Servicio Directo}

Con motivo de clarificar el problema de interés es pertinente mencionar lo siguiente. Al momento existen unas prácticas bélicas en las cuales las fuerzas armadas estadounidenses se encuentran involucradas. Existen estudios que plantean los efectos emocionales y psicológicos que produce haber participado en una guerra y como éstos pueden repercutir en las relaciones de pareja en forma de violencia (Bradley, 2007; McCarroll et al., 2010).

Para entender el efecto que tienen las prácticas bélicas sobre la violencia doméstica de los militares, en el contexto de las fuerzas armadas de los Estados Unidos de América, es 
importante entender lo que significa el despliegue (McCarroll, et al., 2010). Esto resulta necesario ya que el despliegue es una parte esencial del servicio activo militar, entiéndase, la fase en la cual se incurre en prácticas bélicas.

McCarroll y colaboradores (2010) presentan una explicación sobre la posible relación entre el despliegue y la violencia hacia la pareja, lo cual es pertinente al tema bajo estudio. Esto es importante ya que se considera la adaptación y reinserción por parte del veterano así como de su pareja marital. El despliegue se refiere al tiempo en que un miembro de las fuerzas armadas se encuentra separado de su hogar mientras realiza una operación militar. El despliegue tiene tres fases: predespliegue, despliegue y posdespliegue. En el predespliegue el militar entrena para tomar parte en una misión. Esta etapa se caracteriza por la ansiedad ante un despliegue inminente que puede irrumpir el funcionamiento familiar. El despliegue es la etapa en que el sujeto se encuentra sirviendo activamente en una misión bélica. Durante el despliegue, el militar sufre del estrés relacionado con los eventos de la zona de combate y con la preocupación por el bienestar de su familia. Durante el despliegue, la pareja del militar se hace cargo de manejar los asuntos familiares, esto incluye ayudar a sus hijos a lidiar con la ausencia de su padre o madre y la posibilidad de que éste sufra un daño físico o que muera (McCarroll et al., 2010). El posdespliegue se refiere a la etapa en que el militar regresa al hogar. Durante esta etapa la familia debe adaptarse al cambio en la estructura, roles y responsabilidades de la familia, lo que puede ser complejo y provocar ansiedad. Además de las lesiones físicas obvias, en algunos casos las familias militares deben lidiar con las lesiones no aparentes como la depresión, el síndrome de estrés postraumático y la lesión cerebral traumática (Savitsky, Illingworth y DuLaney, 2009). Al ser estos factores de una naturaleza diferente al de la población de 
veteranos en general, el veterano que agrede y que padece de estas condiciones de salud física y mental, requeriría un tipo de intervención diferente a otros agresores, en cuyo historial médico no se encuentran las mismas. Estas situaciones también implicarían trabajar con la familia y los cuidadores.

Una vez el militar de las fuerzas armadas estadounidenses culmina su servicio, se reinserta a la vida civil. Los trabajadores y trabajadoras sociales intervienen con este tipo de situación. Por esto, deben considerar las posibles repercusiones que tiene el haber participado en prácticas bélicas sobre la vida en pareja de los veteranos excombatientes. Es necesario examinar los diversos factores causales de la violencia doméstica y las implicaciones que esto tiene para la práctica del trabajo social en el servicio directo con este grupo poblacional.

Estudiar este tema puede ser de mucho beneficio para los veteranos que incurren en actos de agresión. Quienes cometen actos de violencia hacia sus parejas, experimentan repercusiones a causa de sus propias acciones. Las consecuencias principales son de naturaleza legal ya que la violencia hacia la pareja es un delito grave en Puerto Rico y en otras jurisdicciones (Ley Núm. $54,1989)$. Además de las repercusiones legales se encuentran las repercusiones sociales, tales como el estigma de ser visto como un 'agresor' y repercusiones afectivas, como lo sería la pérdida de una relación.

Cuando un militar activo agrede a su esposa, puede ser expulsado de manera deshonrosa de las fuerzas armadas además de posibles repercusiones penales impuestas por un tribunal militar (6 UCMJ Sec. 928, Art. 128. 1956). Como consecuencia dicho veterano pierde su empleo, los beneficios que recibiese de este, al igual que sus privilegios como veterano. Esto significa que el problema sujeto de estudio puede tener repercusiones sumamente serias para el veterano agresor. 
Otro grupo que se beneficia de los resultados de esta investigación documental es el de las mujeres esposas de veteranos. Éstas son las víctimas o sobrevivientes de la violencia en el hogar. La mujer que vive una situación de violencia en el hogar puede verse afectada física y emocionalmente. Además puede perder la vida si la violencia escala a ese nivel. Cuando la familia tiene hijos e hijas dicha progenie recibe también el impacto de la violencia.

\section{Análisis Histórico de las Manifestaciones de la Violencia Doméstica en los Veteranos}

La violencia doméstica no es un problema nuevo. Lo que "es relativamente nuevo es la denuncia, el estudio y la intervención con esta violencia" (Burgos, 2002, p.27). Esta denuncia comienza en los Estados Unidos a finales de la década del sesenta cuando el movimiento feminista comenzó a luchar porque se reconociera la violencia doméstica "como problema social" (Burgos, 2002, p. 27). Para el año 1975 se celebró en México la Primera Conferencia Internacional de la Mujer, en la cual se expuso el problema social de la violencia doméstica (Burgos, 2002). En el 1993 la organización de las Naciones Unidas presenta una declaración sobre la eliminación de la violencia de Género (Burgos, 2002). En el 1994, la mayoría de los países de la Organización de Estados Americanos (OEA), firma otra medida donde se reconoce la violencia doméstica como "una ofensa en contra de la dignidad humana y como una manifestación de las históricas relaciones desiguales de poder entre los hombres y las mujeres" (Burgos, 2002, p.28). Como resultado de estos movimientos a nivel internacional, los diversos países se movieron a establecer legislación para lidiar con la violencia doméstica. En Puerto Rico estos esfuerzos se 
consolidaron en la formulación de la Ley 54 del 15 de agosto de 1989 conocida como Ley para la Prevención e Intervención con la Violencia Doméstica.

Aunque la violencia es un problema social antiguo, los acercamientos planificados y estructurados para la intervención, son relativamente nuevos. Esto responde a la invisibilidad histórica del problema como se ha expuesto previamente. James y Gilliland (2012), plantean que históricamente el fracaso de los profesionales de ayuda en detectar la violencia de género surge del temor a ofender a los participantes, falta de adiestramiento, falta de tiempo y falta de cubierta médica.

Hay diferentes teorías que explican la violencia doméstica y aportan en alguna medida al estudio y el entendimiento de este fenómeno social. Una de las posiciones planteadas entre los profesionales de la conducta es la teoría feminista. La teoría feminista visualiza la violencia doméstica como determinada por una estructura sexista y patriarcal (Burgos, 2002; Valle Ferrer, 2007; James y Gilliland, 2012). La ideología patriarcal significa la manifestación e institucionalización del dominio de los hombres en la familia y la extensión de ese dominio sobre las mujeres, en la familia y en la sociedad en general.

Si se aplica esta visión a los veteranos excombatientes de las fuerzas armadas de los Estados Unidos que participaron de prácticas bélicas e incurren en violencia doméstica, se debe analizar la violencia machista como mecanismo de control en las fuerzas armadas. Las fuerzas armadas tienen un propósito primario, que es servir a la defensa de la nación. Sin embargo, la historia documentada expone que también han fungido secundariamente como mecanismo de socialización masculina (Molina-Luque, 1992). La socialización militar masculina tiene como objetivo construir el género masculino promoviendo valores estereotipadamente machistas. Algunos de éstos son: 
violencia, fortaleza, dureza, valentía, desprecio a la muerte, entre otros. La internalización de estos valores y creencias tiene dos propósitos. El primer propósito es ser un militar efectivo, entiéndase, que pueda llevar a cabo actos violentos, lo que requiere que se adopte la violencia como algo natural y masculino. El segundo propósito es la obediencia jerárquica. Para ser efectivo, es imperante que el militar acepte ciegamente órdenes de sus superiores. Esa obediencia absoluta se logra con la incorporación de ideales como la patria, la gloria, el honor y el orden. Estos valores se inculcan a través de la violencia. El sujeto militar debe acatar órdenes y asimilar ideales, so pena de castigo mediante actos violentos. Estos actos pueden variar desde insultos verbales, privación de sueño, hasta castigo físico directo. En resumen, se socializa al militar a través de la violencia (Molina-Luque, 1992).

Otras dimensiones a examinar son las consecuencias psicológicas y emocionales de haber participado en conductas bélicas. Este tema se empezó a documentar en el siglo 19 con la guerra civil estadounidense. Documentos de esa época revelan la manera en que se percibían las heridas de combate de naturaleza emocional y psicológica causadas por vivencias traumáticas (Eagan-Chamberlin, 2012). Los militares que sufrían de síntomas relacionados a sus incursiones en prácticas bélicas eran vistos como fracasados que incumplieron con su deber masculino de ser fuertes y valientes. El trauma, y sus repercusiones, significaba, a la luz de la sociedad, que el militar era frágil y carecía de 'hombría' según el concepto socialmente construido (Eagan-Chamberlin, 2012).

Savitsky y colaboradores (2009) plantean que desde el 11 de septiembre de 2001 más de 1.5 millones de tropas han sido desplegadas y muchos de ellos han servido en diversas misiones. Puntualizan estos autores que el personal militar ha 
cambiado y es diverso donde más de 25\% de los militares activos pertenecen a un grupo minoritario. Ellos identifican factores que afectan a la familia de los militares en términos psicosociales. Entre dichos factores se encuentran: largos periodos de separación, problemas de salud física o mental, frecuentes relocalizaciones, muerte y reacciones de los niños a la ausencia del progenitor. Las familias pasan por un periodo de gran estrés y deben luchar con esto, no solamente mientras el militar está activo, sino luego que el veterano se reinserta a la familia y a la vida civil. Todo esto puede tener influencia en los miembros de la familia y cada uno de sus miembros trabajará la adaptación de acuerdo a sus fortalezas personales, familiares y comunitarias.

En el año 2005 en los Estados Unidos hubo cerca de 16,000 denuncias de abuso a la pareja sometidas al Programa de Intercesión Familiar (FAB) (Savitsky et al., 2009). Respecto a la violencia doméstica cometida por veteranos en Puerto Rico, en el año 2013, la Administración de Veteranos en Puerto Rico, recopiló datos a través del programa Veterans Justice Outreach (VJO). Este programa, identifica y brinda seguimiento a través de manejo de casos a los veteranos que enfrentan procesos penales tanto a nivel estatal como federal. Se menciona este programa y las estadísticas recopiladas porque son pertinentes al problema bajo estudio. Los datos recopilados por el programa VJO reflejan que, específicamente para el año fiscal 2009-2010, 18 veteranos fueron acusados formalmente en un tribunal por este tipo de delito; los datos para el año fiscal 2010-2011 reflejan un número de 20 participantes acusados y finalmente se reporta un total de 20 participantes acusados por violencia doméstica para el año fiscal 2011-2012. Es importante señalar que este número podría ser mayor, debido a diversas razones. La violencia de tipo emocional o verbal muchas veces tiende a ser minimizada por la víctima (Molina 
y Fernández, 2009). Se plantea que la mujer maltratada tiende a callar la violencia debido a que no quiere perjudicar al agresor si éste es el padre de sus hijos o el hombre a quien ama (Burgos, 2002). Esto también puede ocurrir, cuando la sobreviviente asocia la violencia con una conducta esperada del género masculino. Al respecto, García Toro (2009) expone que hay una estrecha vinculación entre el género masculino y la violencia mediante el proceso de socialización y formación de género, por lo que la conducta violenta en el hombre es más aceptada socialmente. También recomienda que los trabajadores y trabajadoras sociales analicen y reflexionen sobre "las diversas epistemologías, ontologías y metodologías que los apoya" (p.244).

El estudio de Maldonado-Villafañe (2008) destaca la necesidad de que los veteranos reciban apoyo social y familiar al retornar a la vida civil. Este estudio constó de una muestra de 10 veteranos varones excombatientes que no sufrían de ninguna condición mental. Los sujetos entrevistados presentaron la falta de apoyo familiar y de las instituciones gubernamentales como un asunto que se debe atender. Destacaron la dificultad de conseguir un empleo y la necesidad de mejorar la calidad de los servicios gubernamentales y la falta de calidad en el trato doctor / paciente. Dicho estudio enfatiza el drástico y difícil proceso de re-adaptación así como la necesidad de proveer sistemas de apoyo en la familia y en las instituciones gubernamentales.

El problema planteado refleja la necesidad de implantar un programa de prevención primaria en Puerto Rico, en el cual las familias de los militares conozcan las dimensiones psicosociales de un conflicto bélico para el militar activo, para el veterano que regresa y para la familia. Si existe violencia en la pareja, esto tendrá una repercusión sobre el desarrollo 
psicosocial y el funcionamiento de los hijos e hijas. El impacto será mayor para los menores de cinco años quienes tienen mayores factores de vulnerabilidad (Savitsky, et al., 2009).

A la luz de los factores antes analizados, se podría anticipar que el veterano que regresa al hogar muestre una tendencia a transferir los arreglos estructurales de convivencia de la situación bélica a sus relaciones domésticas y esto puede traducirse a respuestas poco efectivas y rechazadas en el ámbito civil. Bradley (2007), presenta un recuento histórico y los fundamentos teóricos que dirigieron las investigaciones sobre la relación entre la violencia doméstica y el estatus de veterano. Al hacer dicho análisis utilizó la teoría de la influencia cultural. La proposición principal de dicha teoría establece que a mayor aceptación cultural hacia la utilización de la violencia, mayor es la probabilidad de generalizar esta legitimación a otras esferas de la vida donde es menos aceptada socialmente (Bradley, 2007). Se plantea que el escenario militar es una subcultura, la cual es parte y a la vez está separada, de la cultura general de los Estados Unidos de América. A través del proceso de entrenamiento básico los militares son resocializados para acatar las normas, costumbres y valores militares. Dentro de esa socialización se encuentra el entendimiento de que la violencia es a veces necesaria para lograr los objetivos militares. Un concepto militar del ejército de los Estados Unidos conocido como: "mente de batalla" o "battlemind" se refiere a "la fuerza interna del soldado para enfrentar el miedo y la adversidad con valor" (Walter Reed Army Institute of Research, citado en Savitsky, et al., 2009, p. 329). La mente de batalla requiere un nivel de agresión dirigida, que ayuda en el combate, pero resulta negativa en la vida civil del veterano que se reinserta a su vida familiar y a su relación conyugal. Este puede ser un detonante a situaciones de violencia doméstica. 
Hay otros factores sociales que deben tomarse en cuenta al analizar históricamente el desarrollo de la conducta violenta hacia la pareja de los veteranos. Uno de esos factores que ha sido estudiado en relación a la violencia doméstica es el estrés. En la literatura sobre violencia doméstica, algunos investigadores han sugerido que existe una variedad de estresores que pueden tener un efecto detrimental en una relación personal y que al aumentar el estrés podría contribuir a episodios de violencia en una relación íntima (Cano \& Vivian, citado en Bradley, 2007).

El estrés postraumático es un tipo de estrés relacionado a la situación de los veteranos que han incurrido en prácticas bélicas y que constituye, por su impacto, una enfermedad mental. Hoy en día se conoce mucho más sobre el Trastorno del estrés postraumático y sus repercusiones (James \& Gilliland, 2012). Se menciona el desarrollo histórico de dicha condición ya que en el presente hay evidencia documentada sobre su impacto en la vida familiar como consecuencia de conductas violentas exhibidas por el veterano hacia su pareja (Finley, Baker, Pugh \& Peterson, 2010). Además, investigaciones sobre veteranos en combate han demostrado que un veterano generalmente será más agresivo y más propenso a experimentar conflictos maritales si ha estado involucrado en combate durante su servicio militar (Bradley, 2007). Esta puede ser una de las explicaciones de la violencia en las relaciones de pareja de los veteranos excombatientes.

A la luz del análisis realizado se puede concluir que existe la necesidad de capacitar a los trabajadores y trabajadoras sociales en la práctica clínica con esta población, para que puedan entender la etiología de la conducta violenta hacia la pareja de los veteranos que han estado en el frente de batalla. Esto, a su vez, les permitirá desarrollar estrategias de 
prevención y tratamiento. A continuación se presentan algunas recomendaciones a estos fines.

Recomendaciones Específicas para Trabajar con las Necesidades Identificadas

1. Estrategias para atender la necesidad de revisar paradigmas que estudian la etiología y la intervención del trabajo social en servicio directo con veteranos excombatientes que incurren en conducta violenta hacia sus cónyuges o parejas.

1.1 Llevar a cabo actividades profesionales como foros, simposios, conferencias auspiciadas por las instituciones de educación superior en trabajo social, organizaciones profesionales y agencias en el campo del bienestar social, como el Colegio de Profesionales del Trabajo Social, la National Association of Social Workers, la Oficina de la Procuradora de las Mujeres, el Departamento de Justicia, el Departamento de Corrección, el Departamento de la Familia, la Oficina del Gobernador así como agencias de base comunitaria. El objetivo de esta estrategia será que cada organización presente su visión sobre el problema de la violencia doméstica y los programas de intervención directa con el agresor. De estas actividades podría surgir un equipo de trabajo compuesto por representantes de los diversos sectores representados. Las presentaciones producto de estas actividades se recopilarían en documentos que podrían incorporarse a los cursos de trabajo social y de otras disciplinas. También podrían dar lugar a investigaciones sobre este tema que ayuden 
a apoyar las mejores prácticas basadas en evidencia.

1.2 Hacer pública la información relacionada a los programas de intervención con personas con conducta violenta hacia sus parejas. Esta información debe contener procedimientos, métodos de intervención y resultados de investigaciones y evaluaciones, si alguna.

1.3 Hacer públicas y accesibles las estadísticas de violencia doméstica a los usuarios de internet. Es necesario crear un banco de datos que facilite las investigaciones, ya que muchas veces las estadísticas y datos disponibles no están actualizados y se observa cierta resistencia al compartir la información. En relación a los veteranos esto es aún más complejo.

2. Estrategias para atender la necesidad de impactar a través de la educación, la prevención y la intervención en trabajo social al veterano con conducta violenta hacia su pareja.

2.1 La Administración de Asuntos al Veterano podría diseñar un programa específico de prevención de la violencia doméstica para los veteranos. Se puede establecer un procedimiento estándar con todos los veteranos que solicitan los servicios, donde se explore si existen indicadores de conducta violenta en la pareja. Esto podría contribuir a que se pudieran identificar situaciones en una etapa tempana.

2.2 Establecer un programa de orientación estándar sobre aspectos sociales, psicológicos y legales 
de la violencia doméstica, para todos los militares que se retiren del servicio activo. Esta medida podría contribuir a fortalecer las capacidades adaptativas del veterano y su respuesta a situaciones de estrés en sus transacciones con las demandas del ambiente civil en la familia y en la comunidad.

2.3 Establecer un programa de orientación estándar sobre aspectos sociales, psicológicos y legales de la violencia doméstica, para todos los veteranos que comiencen a trabajar en un empleo. Esta medida podría contribuir a fortalecer las capacidades adaptativas del veterano y su respuesta a situaciones de estrés en sus transacciones con las demandas del ambiente civil en el empleo.

2.4 Establecer un programa de desvío en la Administración de Asuntos del Veterano. Se recomienda un enfoque basado en una perspectiva de fortalezas y enfocado en soluciones (Lehman y Simmons, 2009). Esta estrategia es factible, ya que la agencia cuenta con los recursos económicos y humanos necesarios para establecer dicho programa. El autor tuvo la oportunidad de diseñar e implantar junto a otra trabajadora social de la Administración de Veteranos en Puerto Rico, un grupo de intervención directa en trabajo social para veteranos con casos legales (Fernández-Lizardi y Rivera, 2013). El mismo se realizó como Proyecto Social y parte del internado del que suscribe. La intervención fue dirigida por una perspectiva de 
fortalezas y el modelo de intervención grupal enfocado en soluciones según presentado por Lee, Uken y Sebold (2009).

Al finalizar el grupo se llevó a cabo una evaluación cualitativa de la experiencia, donde se encontró que los participantes del grupo percibían que habían logrado cambios en conducta hacia su pareja y en su motivación para mantener dicha conducta. La misma fue grabada mediante el consentimiento informado de los participantes y está en disposición de la Administración de Veteranos. En uno de los casos, hubo el testimonio de la esposa de uno de los participantes confirmando el cambio de su pareja. Una de las observaciones de los veteranos fue que les había satisfecho y provisto esperanza el que este grupo se dirigiera al presente y al futuro, a diferencia del enfoque de otros grupos terapéuticos de los cuales habían participado.

Los grupos de resocialización y reeducación muchas veces se basan en técnicas de confrontación, por lo cual el participante trata de expresar la conducta que cree esperan los profesionales (Gondolf, 2012). Tener un programa de desvío dirigido a los veteranos puede servir a obtener resultados más confiables. Al tener estos programas, se puede obtener información de primera mano sobre las necesidades del veterano y proveer un mejor servicio.

3. Estrategias para atender la necesidad de llevar a cabo estudios de investigación para documentar la efectividad de los programas dirigidos a la reducación 
de los hombres que expresan violencia y han sido convictos por la Ley 54.

3.1 A través de esta investigación documental se evidenció la carencia de investigaciones que fundamentaran las mejores prácticas de intervención con el veterano excombatiente que incurre en conducta violenta hacia su pareja. Aún la investigación en los Estados Unidos está llena de interrogantes y puntos en divergencia. Gondolf (2012) llevó a cabo un análisis de las opiniones de diversos directores de los programas de reeducación encontrando opiniones diversas y hasta contradictorias de los mismos. Molina, Fernández y Berríos (2015) llevaron a cabo un estudio cuantitativo sobre la efectividad de los servicios para agresores y agresoras de violencia doméstica. La muestra estuvo constituida por 84 personas egresadas de los servicios ofrecidos por el Proyecto de resocialización masculina y femenina, auspiciado por el Programa AFANA en Puerto Rico durante los años 2004-2014. En dicho estudio se encontró que un $96 \%$ de los egresados, no se había involucrado en actos de violencia hacia su pareja, después de recibir los servicios. Cabe destacar que solamente el $40 \%$ de las personas había tenido una orden de protección al entrar al programa de servicios, el cual no es un programa de desvío sino de prevención a nivel secundario (Molina, Fernández y Berríos, 2015). No obstante, el estudio no estaba dirigido a veteranos sino a la población general. $\quad$ En torno a los programas de tratamiento para agresores Gondolf (2012) 
recomienda que se lleven a cabo estudios sobre la efectividad de los programas con hombres que maltratan a su pareja, donde se utilicen tanto enfoques experimentales como enfoques de tipo cualitativo, tales como: grupos focales, observación clínica, investigación participativa, entre otros.

Esta estrategia es factible a través de las diversas instituciones de educación superior y las escuelas y programas graduados de trabajo social. Se pueden llevar a cabo trabajos de investigación, tesis de maestría y disertaciones doctorales en trabajo social, relacionados al trabajo social con veteranos y la violencia doméstica. Además la academia puede estimular que la facultad también se involucre en la investigación, apoyando estos esfuerzos. Por otro lado, la Administración de Asuntos del Veterano en Puerto Rico tiene recursos humanos con preparación en investigación los cuales al unirse a los trabajadores sociales en el servicio directo, pueden abordar dichos temas. Estas estrategias son viables y pueden contribuir grandemente a la investigación y al servicio directo del trabajo social con la población de veteranos.

4. Estrategias para lidiar con la necesidad de revisar currículos de trabajo social en Puerto Rico y ofrecer cursos específicos o concentraciones en el trabajo social con militares y veteranos.

4.1 Esta investigación documental evidenció la necesidad de revisar y fortalecer la práctica del trabajo social con militares y veteranos. La educación formal sobre la violencia doméstica 
puede tener efecto sobre la comprensión de este problema y la eficacia del personal de servicio. Esta fue una conclusión del estudio sobre la comprensión de la violencia de parejas, de los proveedores de servicios de salud mental de la Oficina de Asuntos del Veterano (Edwardsen et al., 2011). Los Programas Graduados en Trabajo Social de la Universidad Interamericana y la Universidad de Puerto Rico tienen acuerdos colaborativos con la Administración de Veteranos de Puerto Rico. Los estudiantes tienen la oportunidad de realizar su internado en dicha agencia, la cual provee un excelente escenario de aprendizaje a sus estudiantes. Sin embargo, sus respectivos currículos no cuentan con un sólo curso de especialidad en la intervención en servicio directo con los veteranos.

Ambos programas podrían estudiar el interés de sus egresados y de sus estudiantes prospectivos en trabajar con los veteranos y sus familias. Esto podría dar base a la creación de un currículo que tenga este enfoque. Puede ser un currículo completo, una sub especialidad dentro de las concentraciones o una certificación luego del grado de maestría. Para esto se puede llevar a cabo una encuesta con el personal docente del Hospital de Veteranos para determinar cuáles son las áreas de necesidad que ellos han observado en los estudiantes que realizan su internado en dicha agencia. Existe la necesidad de que estos estudiantes sean adiestrados para poder intervenir en forma temprana con asuntos del veterano tales como: intercesión familiar y 
asuntos relacionados a la violencia doméstica, abuso de drogas y alcohol, estrés postraumático, abuso sexual y suicidio.

Entre los cursos que deberían crearse estarían:

- $\quad$ Salud mental en el trabajo social con los militares y veteranos

- Intervención en crisis con militares, veteranos y sus familias

- Intervención en trabajo social con veteranos con problemas de abuso de sustancias

- $\quad$ Investigación en asuntos del militar y el veterano

- $\quad$ Práctica avanzada con veteranos

- $\quad$ Trabajo social con situaciones de violencia familiar en familias de veteranos

- $\quad$ Seminario integrador en asuntos del veterano

Los trabajadores y trabajadoras sociales en la práctica directa de trabajo social con militares y veteranos, la National Association of Social Workers y el Colegio de Profesionales del Trabajo Social en Puerto Rico pueden ser recursos para propiciar el adiestramiento de los profesionales que intervienen con esta población. La academia podría contribuir con su conocimiento a fortalecer la intervención basada en evidencia y promover la investigación sobre los militares y veteranos, sus diversas situaciones de vida, incluyendo un problema tan antiguo y complejo como la violencia en la relación de pareja. 


\section{Referencias}

Bradley, Christopher (2007). Veteran Status and Marital Aggression: Does Military Service Make a Difference? Journal of Family Violence, 22 (4), 197-209.

Brands, Henry W. (2012). What do we owe our veterans? American History, 47 (2), 68-73.

Burgos, Nilsa, M. (2002). Violencia en la relación de pareja: Un desafío en la promoción de salud y prevención de la enfermedad. Revista ANALISIS, III (1), 28-46.

Corsi, Jorge (1995). Violencia masculina en la pareja: Una aproximación al diagnóstico ya los modelos de intervención. Buenos Aires: Paidós.

Eagan-Chamberlin, Sheena, M. (2012). Emasculated by trauma: A social history of post-traumatic stress disorder, stigma, and masculinity. Journal of American Culture, 35 (4), 358-365.

Edwardsen, Elizabeth A., Dichter, Melissa E., Walsh, Peter \& Cerulli, Catherine (2011). Instructional curriculum improves medical staff knowledge and efficacy for patients experiencing intimate partner violence. Military Medicine, 176 (11), 1260-1264.

Finley, Erin, Baker, Monty, Pugh, Mary Jo \& Peterson, Alan (2010). Patterns and perceptions of intimate partner violence committed by returning veterans with post-traumatic stress disorder. Journal of Family Violence, 25 (8), 737-743.

Fernández-Lizardi, Antonio \& Rivera, Ghislaine (Producers) (2013). Taller de relaciones interpersonales positivas (TRIP): A focus group evaluation of a social group work intervention with participants of the Veterans Justice Outreach Program \{DVD\}. San Juan, PR: Veterans Administration Health Center. 
García Toro, Víctor I. (2009). Reflexiones en torno al género masculino: Violencia y trabajo social en el contexto penitenciario. Revista ANALISIS, XX (1), 243-267.

Gondolf, Edward W. (2012). The future of batterer programs: Reassessing evidence-based practice. Boston, MA: Northeastern University Press.

Maldonado-Villafañe, Milagros (2008). La percepción del veterano puertorriqueño sobre su experiencia bélica y la reinserción en la vida civil. Disertación doctoral no publicada, Universidad Interamericana, San Juan, Puerto Rico.

James, R. \& Gilliland, B. E. (2012). Crisis intervention strategies. Belmont, CA: Thomson.

Lehmann, Peter \& Simmons, Catherine A. (2009). Strengthsbased batterer intervention: A new paradigm in ending family violence. New York: Springer Publishing Co.

Lee, Mo Yee, Uken, Adriana \& Sebold, John (2009). Accountability for change: Solution focused treatment of domestic offenders. In Lehmann, P. \& Simmons, C. A. (Eds.) Strengths-based batterer intervention: A new paradigm in ending family violence (pp. 55-85). New York: Springer.

Ley de Prevención e Intervención con la Violencia Doméstica, Ley Núm. 54 de 15 de agosto de 1989, según enmendada, 8 L.P.R.A. sec. 601.

Maschi, Tina, Bradley, Carolyn \& Ward, Kelly (2009). Forensic social work: Psychosocial issues in diverse practice settings. New York: Springer Publishing Company.

McCarroll, James. E., Ursano, Robert. J., Xian, Liu, Thayer, Laurie. E., Newby, John H., Norwood, Ann E., \& Fullerton, Carol S. (2010). Deployment and the Probability of Spousal Aggression by U.S. Army Soldiers. Military Medicine, 175 (5), 352-356. 
Molina-Castro, Gloria M., \& Fernández, Antonio (2009). Perfil del agresor y algunos procesos de la intervención grupal. Scientific International Journal, 6 (1), 15-37.

Molina-Castro, Gloria M., Fernández, Antonio, \& Berríos, Yarisel (2015). Efectividad de los Servicios Socio-Educativos a Agresores de Violencia Doméstica a través del Proyecto de Resocialización Masculina y Femenina. Scientific International Journal, 12 (2), 5-18.

Molina-Luque, Fidel (1992). Quintas y servicio militar: Aspectos sociológicos y antropológicos de la conscripción (Lleida, 1878-1960). Servei de Publicacions: Universitat de Lleida, 54-98.

Rosa Soberal, Rosalie (Ed.) (2007). La diversidad cultural. San Juan, PR: Publicaciones Puertorriqueñas.

Savitsky, Laura, Illingworth, Maria, \& DuLaney, Megan (2009). Civilian Social Work: Serving the military and veteran Populations. Social Work, 54 (4), 327-339.

Uniform Code of Military Justice, 6 UCMJ Sec. 928, Art. 128. (1956).

Universidad Interamericana, Escuela Graduada de Trabajo Social (2013). Competencias y conductas prácticas de la concentración de servicio directo. Manuscrito sin publicar. San Juan, PR.

Universidad Interamericana de Puerto Rico (2009-2011). Catálogo Graduado. San Juan, PR.

Universidad de Puerto Rico, Recinto de Río Piedras, Decanato de Estudios Graduados e Investigación (2009-2011). Catálogo Graduado. San Juan, PR. 
Valle Ferrer, Diana. (2006). Estrategias de enfrentamiento y resistencia en mujeres sobrevivientes de violencia doméstica en Puerto Rico. Revista ANALISIS, VII (1), 37-70.

Valle Ferrer, Diana (2007). La opresión contra las mujeres en la familia: violencia doméstica y resistencia. En: Rosa Soberal, R. (2007). La diversidad cultural: reflexión crítica desde un acercamiento interdisciplinario. San Juan, PR: Publicaciones Puertorriqueñas, 247-270.

Veterans Administration (2013). Veterans Justice Outreach Statistical Report. San Juan, Puerto Rico.

Webster's New World Dictionary and Thesaurus (1996). New York: Simon \& Schuster, Inc. 